\title{
Effect of eye movements on backward masking and perceived location*
}

\author{
MICHAEL L. DAVIDSON. MARY.JUNE FOX. and A. O. DICK' \\ iniversity of Rochester. Rochester. . Vew York 14627
}

\begin{abstract}
A typical trial of this masking experiment involves. in quick succession. presentation of five letters. evocation of an eve movement. and presentation of a spatially localized mask. either a visual-pattern mask or a metacontrast ring. The effect of the mask is to suppress the report of the letter that stimulates the same retinal location, even though the mask appears to cover or surround the letter whose position in real space it shares. Masking is, however. weaker when the eyes move than when they do not. An auxiliary experiment suggests that the spatial aspects of observable (reportable by S) stimulus persistence are unaffected by eye movements, and therefore that observable persistence differs from that susceptible to masking.
\end{abstract}

The phenomenon of backward masking implies that some representation of the test stimulus is preserved until the subsequent mask stimulus can interact with it. The possible function of this short-term visual memory: has intrigued information-processing theorists (e.g., Neisser, 1967). Leading to the suggestions (1) that this memory is the same "iconic" representation revealed in delayed-partial-report experiments (Sperling, 1960; Averbach \& Coriell. 1961), (2) that this memory accounts for apparent stimulus persistence (Haber \& Standing. 1969). and (3) that the preservation of the stimulus gives recognition processes time to work.

Outside the laboratory: a sudden change in retinal stimulation is commonly the result of an eye movement. It is therefore important to know the effect of an eye movement upon the short-term stimulus representation. Conceiving this representation as iconic suggests three possibilities. First, the representation may behave as a retinal afterimage, moving with respect to distal space with the eyes, in which case stimuli from successive fixations would tend to mask each other. Second, the representation may be so altered by an eye movement that it moves with respect to the retina, as would a stimulus persisting in the visual field. In this event, stimuli from successive fixations might be mutually supportive, perhaps, in Neisser's (1967) terms, snapshots of various portions of the visual field that can be synthesized into a single visual percept. Such a representation would also account for the observed correlation between postexposure eye movements and locus of most accurate report (Bryden, 1961; Crovitz \&

*Supported by the U.S. Public Health Service, Research Grant 1-R03-MH20911 to M.L.D., and by the U.S. Office of Education, Grant OFG-2-710371B to A.O.D. Opinions expressed do not necessarily reflect the position or policy of the Office of Education and no official endorsement should be inferred.

$\div$ Requests for reprints should be sent to one of the authors at the Department of Psychology, University of Rochester. Rochester, New York 14627. Several valuable suggestions by Robert M. Boynton are gratefully acknowledged. The help of Geoffrey Inglis in the design and construction of special electronic equipment is also much appreciated.
Davies, 1962). Third, the eye movement may itself affect stimulus persistence, either by suppression of the premovement stimulus or by influencing the interaction between pre-and postmovement stimuli.

\section{MASKING IN THE PRESENCE OF EYE MOVEMENTS}

Suppose that a stimulus flash consisting of five letters in a horizontal row is given, then S's eyes move two letters to the left, and finally a visual-pattern mask is flashed that covers the third letter in the actual visual field and therefore stimulates the same retinal region as the fifth letter. The question is, if the mask has an effect, will it be upon the fifth letter or the third letter? If it is the fifth, masking may be called "afterimage-like," if the third, "percept-like." since, as established in the succeeding section, the mask would then suppress the letter whose location it is (correctly) perceived to share.

\section{Stimuli}

A tachistoscope (Gerbrands Model T-3B-1C, with lamp drivers. Model 400-3), modified to provide fixation lights in a fourth channel, was used to present stimuli whose spatial arrangement is shown in Fig. 1. Five letters constituted each test stimulus and were drawn at random without replacement from the 20 letters remaining when AEIOL and $\mathrm{J}$ were excluded from the alphabet. Each mask stimulus was either one pattern mask or one metacontrast circle, appearing subsequent to the test stimulus at one of the five letter locations. The temporal sequence of stimuli was: blank background field until the start of the trial, 10-msec test stimulus, background field for a specified interstimulus interval (ISI), 60-msec mask, background field. On no-mask control trials, the flashed mask field was blank. The luminance of the mask and background fields was about $3 \mathrm{~mL}$; that of the test stimulus was about $1 \mathrm{~mL}$. slightly dimmer to achieve less-than-perfect visibility. Viewing was free and binocular, with head position controlled by chin- and foreheadrests. One of the two fixation lights was visible at any moment. appearing as a yellow filament, bright enough only to be easily seen, and positioned just below the second or fourth letter. 

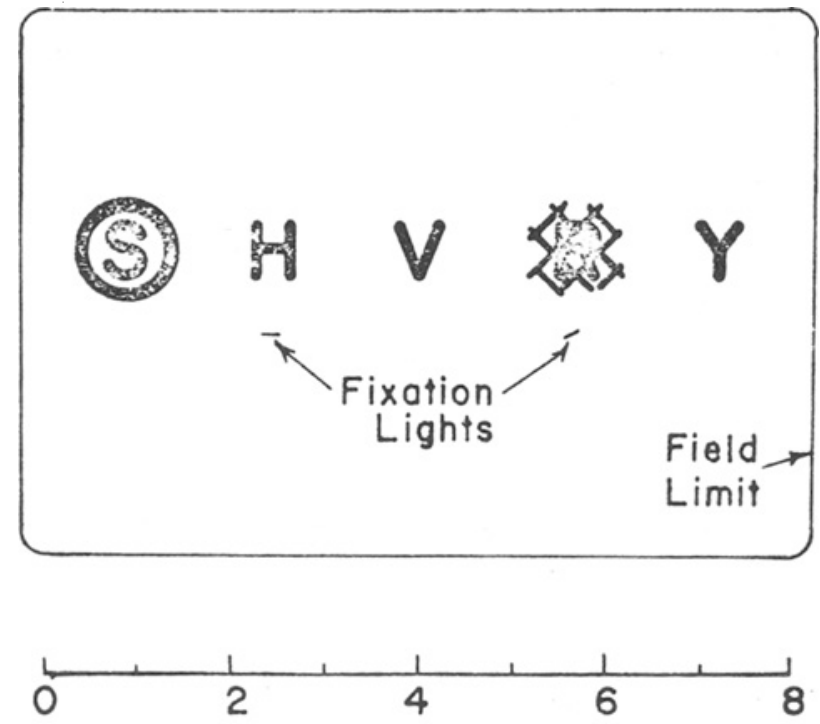

Degrees of Visual Angle

Fig. 1. Spatial arrangement of stimuli, masking experiments. $S$ in Position 1 surrounded by typical metacontrast circle; $R$ in Position 4 covered by typical pattern mask.

\section{Baseline Experiments}

To insure that the mask has a local effect and to

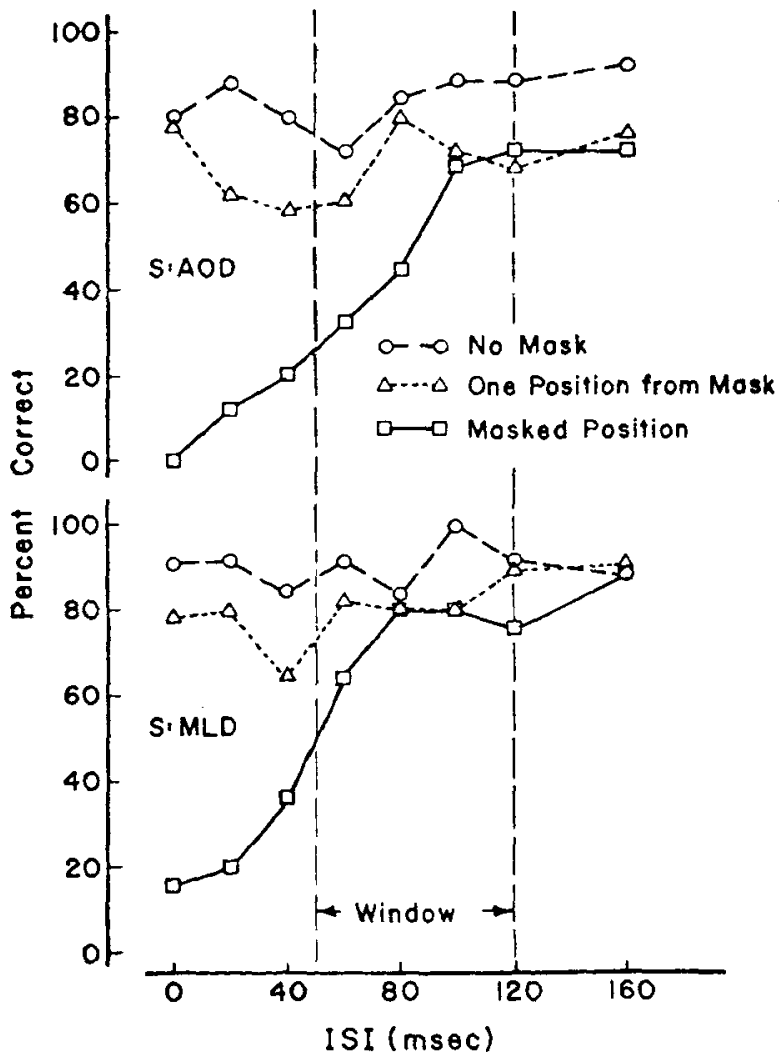

Fig. 2. Results of baseline experiment with visual pattern mask. determine the range of delays at which masking occurs, control experiments were carried out on both pattern and metacontrast masks.

\section{Method}

Each trial was under the control of the tachistoscope timer (Gerbrands Model 300-6L). The S was to fixate upon the left fixation light, the only one illuminated, and to report the five letters and the location of the mask, if any. ISI was varied among the eight values of $0,20,40,60,80,100,120$, and $160 \mathrm{msec}$, and each type of mask was presented in six conditions, i.e., the five positions and no mask. The $S$ experienced, once in each 48-trial session, all combinations of ISI and mask condition in a random order; five sessions for each type of mask and each of two Ss constituted the experiments.

\section{Results}

The percentage of letters correctly reported is presented in summary form in Fig. 2 (visual pattern mask) and Fig. 3 (metacontrast circle). The two Ss appear to differ; the data are therefore presented separately. The no-mask data summarize all reports given with the blank "mask," and indicate about 90\% visibility of the letters. The masked-position data include

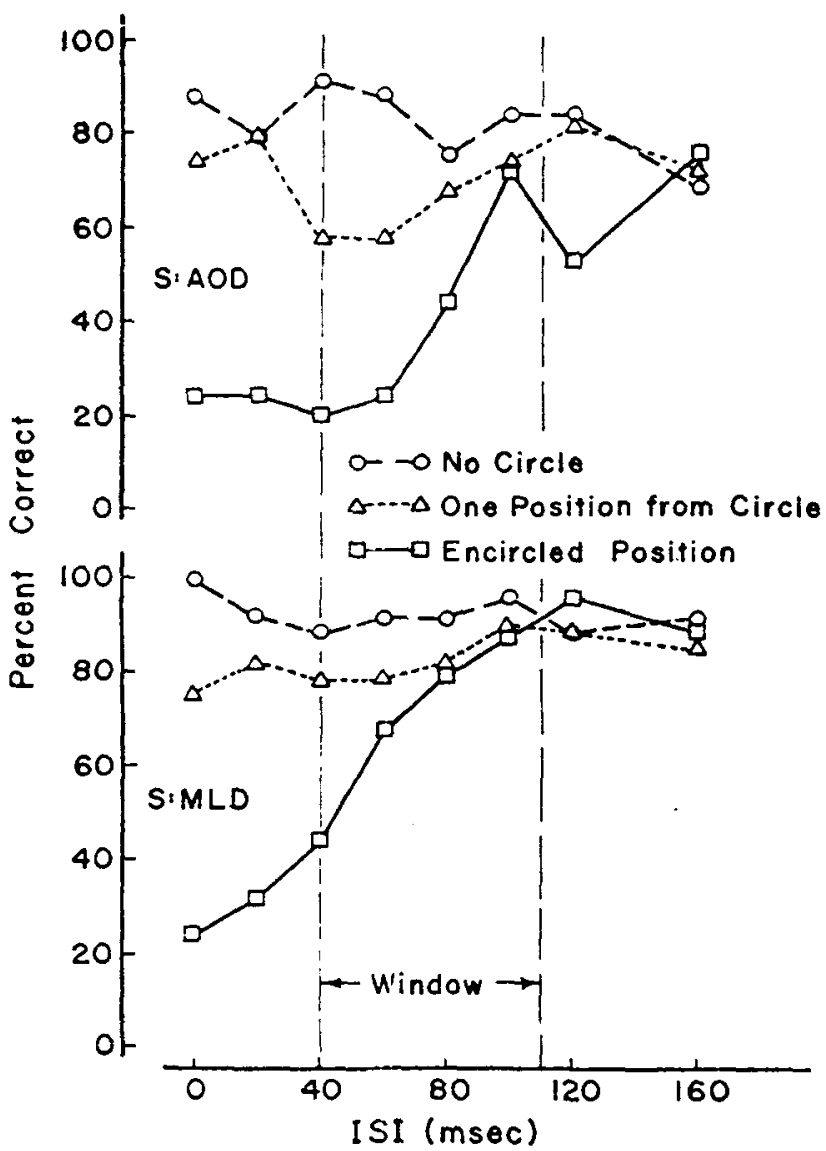

Fig. 3. Results of baseline experiment with metacontrast circle. 
from each trial the report of just that letter spatially contiguous with the subsequent mask: the one-position-from-mask data summarize the reports of the letters adjacent to the masked letter. In the last case. the ordinate is average percent correct, the average being taken over letter positions equally weighted (the five averaged percentages are: percent correct report of Letter 1 when mask is in Position 2, percent correct report of Letter 2 when mask is in Position 1 or in Position 3, etc.). Thus. the usual letter-position effects were averaged equally for all three curves. The "windows" indicated pertain to the eye-movement experiments.

Three aspects of these results are noteworthy: Masking (1) is strongly dependent on ISI, (2) is mainly confined to the letter covered or surrounded by the mask. and (3) apparently affects letters one position removed to some degree. The results generally agree with other data on metacontrast masking under comparable circumstances (Averbach \& Coriell, 1961; Eriksen, Collins. \& Greenspon. 1967), and indicate that the pattern mask has much the same effect (cf. Smith \& Schiller. 1966).

\section{Eve-Movement Experiments}

\section{Evocation of Eye Movement During the ISI}

The baseline-experiment data make it clear that, to investigate the effect of a post-eye-movement mask upon a pre-eye-movement stimulus, the eye movement must be completed as soon after the stimulus as possible, not later than about $120 \mathrm{msec}$. This was accomplished (1) by evoking a regular pattern of eye movements and synchronizing the letter stimulus with it, and (2) by causing the eye movement to trigger the mask.

Two German-silver disk electrodes affixed to S's temples and a reference electrode on the forehead provided an electro-oculogram (EOG) signal reflecting the summed rotation of the two eyes. Grass Model 7 differential amplifier and chart recorder presented the EOG signal for observation by $E$ and for triggering the mask.

An experimental (eye-movement) trial occurs as follows: The two small fixation lights are alternately illuminated for $650 \mathrm{msec}$. The $S$ follows the light with his eyes, and $E$ watches the amplified EOG signal on the chart recorder. When satisfied with the signal, E presses the start switch, causing the timer to begin with the next alternation of the fixation light. After a preset delay, the letter stimulus is presented. Meanwhile, a gate has been opened so that the next eye movement in the appropriate direction triggers the mask-stimulus circuitry. The mask is delayed by $25 \mathrm{msec}$ after the EOG trigger (to permit the eye movement to complete its course) and then the mask is presented. The amount of time between stimulus and mask initiations (stimulus-onset asynchrony, SOA) is available on an

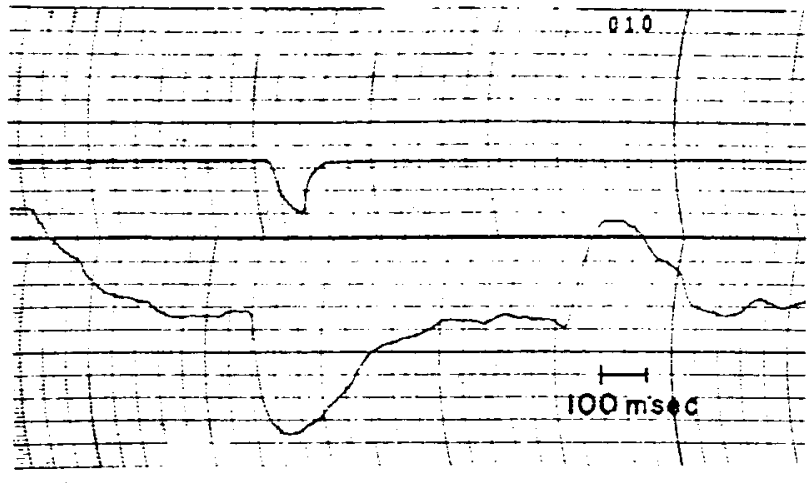

Fig. 4. Typical triggering of mask (upper record) by EOG signal (lower record). Upper record is damped; mask is on for $60 \mathrm{msec}$ with fast rise and fall speeds. EOG is ac-coupled to trigger and to record.

Electronics Research Corporation Model 2623 gated clock. To present a control (no eye movement) trial, the fixation-light alternator is turned off, leaving one of the lights steadily illuminated. The stimulus and mask are presented in a sequence determined entirely by the tachistoscope timer.

A typical record of the EOG signal and triggered mask is shown in Fig. 4. The position of the eyes is shifting from one side to the other about every $650 \mathrm{msec}$. The signal shown (and used in triggering) is bandpass filtered: the unfiltered signal revealed that the eye movement required no more than $35 \mathrm{msec}$. The mask began $35.40 \mathrm{msec}$ after the onset of the eye movement.

The stimulus/eye-movement interval was not as readily controlled as the eye-movement/mask interval, since the former depended on the synchrony between the eye movement and the movement of the fixation light. Only ISIs within a $70 \cdot \mathrm{msec}$ range, or window (Figs. 2 and 3 ), were permitted. Three steps were taken to ensure that each ISI was, in fact, within the window: (1) In preliminary trials, $\mathbf{S}$ was told to follow the fixation light, and to report the letters, etc., as in the experiment proper. The $\mathrm{E}$ recorded the stimulus-onset asynchrony (SOA = ISI plus $10 \mathrm{msec}$ ) for each of about 20 trials. The stimulus-delay interval was then adjusted so that S's typical ISI fell in the middle of the window. (2) In the experiment itself, S was informed of the SOA after each trial, so that he could attempt to adjust subsequent performance if necessary to hit the window. (3) Data were collected only from those trials with acceptable ISI; other trials were repeated later in the session (with new letters each time), until an acceptable ISI was achieved. The eye-movement task was not easy for S: 20 -trial hit rates for the window ranged from $30 \%$ to $95 \%$. Overall, somewhat less than half of all trials had to be repeated.

\section{Method}

Design. For each mask type, the eye-movement experiment consisted of eight types of trial, defined by three two-level 
Table 1

Data Collected in Eye-Movement Masking Experiments

\begin{tabular}{cccccc}
\hline & \multicolumn{2}{c}{ Pattern Mask } & & \multicolumn{2}{c}{ Metacontrast Ring } \\
\cline { 2 - 3 } \cline { 5 - 6 } S & $\begin{array}{c}16- \\
\text { Series* }\end{array}$ & $\begin{array}{c}\text { Addi- } \\
\text { tionali }\end{array}$ & & $\begin{array}{c}16- \\
\text { Series* }\end{array}$ & $\begin{array}{c}\text { Addi- } \\
\text { tional } †\end{array}$ \\
\hline A.O.D. & Yes & No & & Yes & 2 Series \\
M.L.D. & Yes & 4 Series & & Yes & 4 Series \\
S.H.B. & Yes & No & & No & No \\
H.G. & No & No & & Yes & 4 Series \\
\hline
\end{tabular}

*320 trials, all experimental conditions

†20 trials per series, all with eye movements and masks

factors: (1) eye movement vs control (whether an eye movement intervened between stimulus and mask). (2) mask present vs blank masking field, and ( 3 ) location of fixation during stimulus (left-under second letter; right-under fourth). (Observe that an eye-movement trial with fixation on the left involves a right-going eye movement between the stimulus and mask.) Experimental trials were presented in 20 -trial series. The number of series run for each $\mathrm{S}$ is shown in Table 1 .

For each type of mask and each $S$, the major part of the experiment involved 16 series, 4 of each combination of fixation location and eye movement or control. Each series contained 10 trials with masks -2 in each of the five possible locations - and 10 without masks. The 20 trials of each series were ordered randomly; the 16 series occurred in an order that counterbalanced the four conditions. Because the amount of masking is strongly dependent on ISI, even within the window (Figs. 2 and 3), ISIs for the control series were yoked to eye-movement ISIs: The 20 observed (and acceptable) SOAs from each eye-movement series were set on the timer for the 20 trials of a subsequent control series. When the counterbalancing required that a control series begin the experiment. it was yoked to preexperiment practice eye-movement trials. Thus, the distribution of ISIs within the window was essentially the same for each S'scontrol and eye-movement trials.

Additional series were presented to the $S$ s as convenient and as indicated in Table 1: the additional data concerned only masking with eye movements, and were collected in the vain hope of obtaining clear results from each $\mathrm{S}$ separately. These additional data are included in the results here reported.

Procedure. Before each eye-movement series, S was asked to follow the alternating light and the EOG amplifier was adjusted for best triggering. A few warm-up trials were then given, followed by as many trials as needed to yield 20 acceptable ISIs. The S's task on each trial was to follow carefully the fixation light, to report as many of the five letters as possible, and to report the location of the mask, if any. Eye movements subsequent to the mask could not be adequately recorded with the apparatus used; informal observation suggests that the stimulus presentation did disrupt the eye-movement pattern on occasion. The same procedure was used on control trials, except, of course, that $S$ maintained steady fixation and received no report from $\mathrm{E}$ about his ISI.

Collation of data. Each trial was scored without regard for order of report, i.e., by determining whether each letter presented was among the letters reported by $S$. The data for all the trials in a given condition (e.g., eye movements, fixation on left, pattern mask) were collated on the basis of letter position relative to the mask, to yield percent correct report. For example, the result for the letter two positions to the right of the mask is based on the report of (a) the third letter when the mask is in Position 1. (b) the fourth letter with the mask in Position 2, and (c) the fifth letter with the mask in Position 3. Trials with no mask were, for the purpose of scoring, assigned virtual-mask locations. in an effort to equate letter-position effects in the collated mask and no-mask data. All relevant available data were included in the collation for each $\mathrm{S}$ and each of the experimental conditions. In particular, (1) main- and additional-series data were combined in each eye-movement mask condition, and (2) for A.O.D. and M.L.D., no-mask data from the pattern-mask experiment were pooled with those from the metacontrast experiment.

\section{Results}

The effect of the mask upon the report of letters is summarized in Figs. 5 and 6. Each figure is based on data from three Ss, as indicated in Table 1. From the information in the preceding sections, it can be reckoned that the total number of trials per data point ranges from 64 (Fig. 5, control, L-M distance of 4) to 420 (Fig. 6, eye movement, L-M distance zero); observe, however, that the error markers reflect variance among the three $\mathrm{Ss}^{\prime}$ means. The control-condition data reinforce the conclusion from the baseline experiments, that masking is primarily of the letter masked and secondarily of neighboring letters. The eye-movement data indicate (1) that the effect of a mask is upon the letter that occupies the same retinal location-that

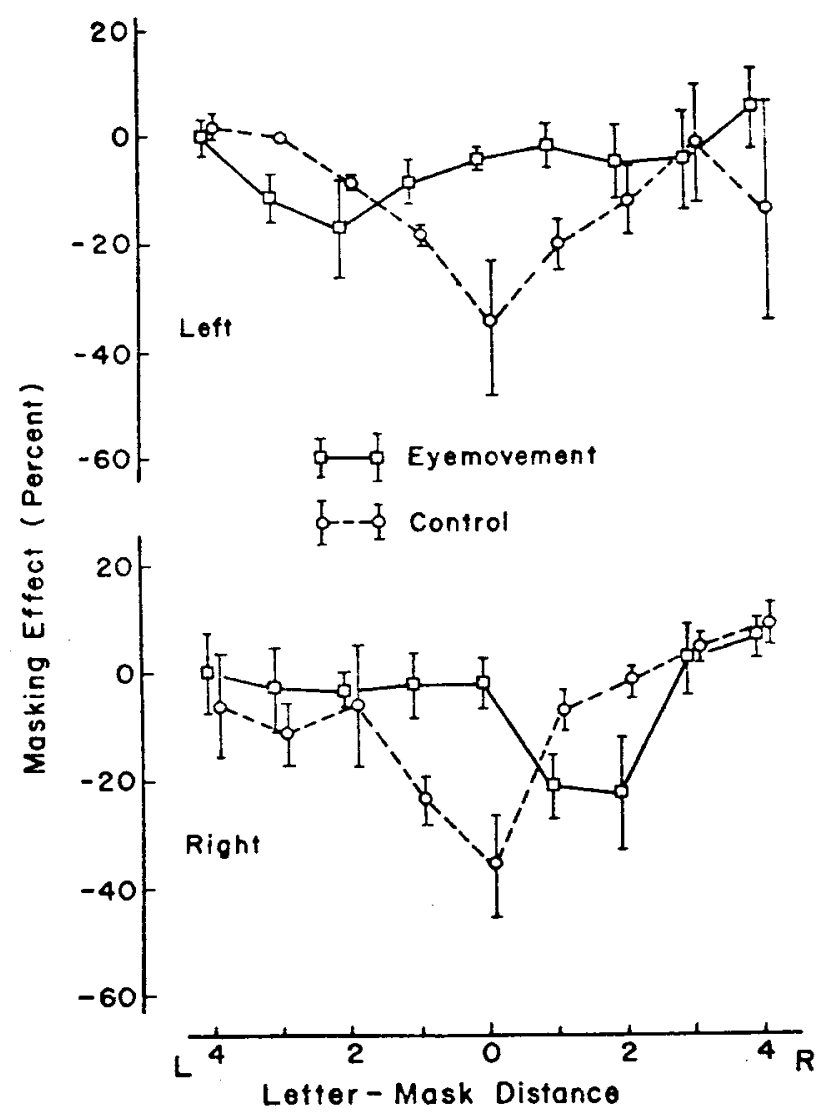

Fig. 5. Results of eye-movement experiment with visual pattern mask. Percent correct with mask minus percent correct with no mask (masking effect) as a function of letter position relative to mask. Poin ts-mean of three $S s$; bars - \pm 1 standard error of the mean. Upper curves-left fixation on letters; lower curves-right fixation on letters. 
masking is afterimage-like, and (2) that masking is weaker in the eye-movement condition than in the control. The following statistical tests confirm these impressions: By pooling noise and metacontrast data (S.H.B. and H.G. are conservatively taken as one S), each $\mathrm{S}$ was given a percent-masking score for each of the four combinations of eye movement vs control and left vs right: data were considered for only the letter that occupied the same retinal position as the mask. Inference to the population of all Ss is accomplished for any specified combination of these four numbers by a $t$ test with $2 \mathrm{df}$. Such tests revealed significant average masking in the two ( $\mathrm{L}$ and $\mathrm{R}$ ) eye-movement conditions $(\mathrm{t}=2.93, \mathrm{p}<.05$, one-tailed $)$, and in the two control conditions $(t=6.14, p<.05$, one-tailed $)$; moreover, average eye-movement masking is significantly weaker than average control masking $(t=9.92, p<.05$, two-tailed). Similarly significant results are obtained upon examining total masking effect over all letters rather than the retinally coincident one. Descriptively, masking with eye movements is about half that without: the average amount of retinally coincident masking in all the eve-movement data is $54 \%$ of that in all control data: eye-movement masking over all letters is $41 \%$ of control masking.

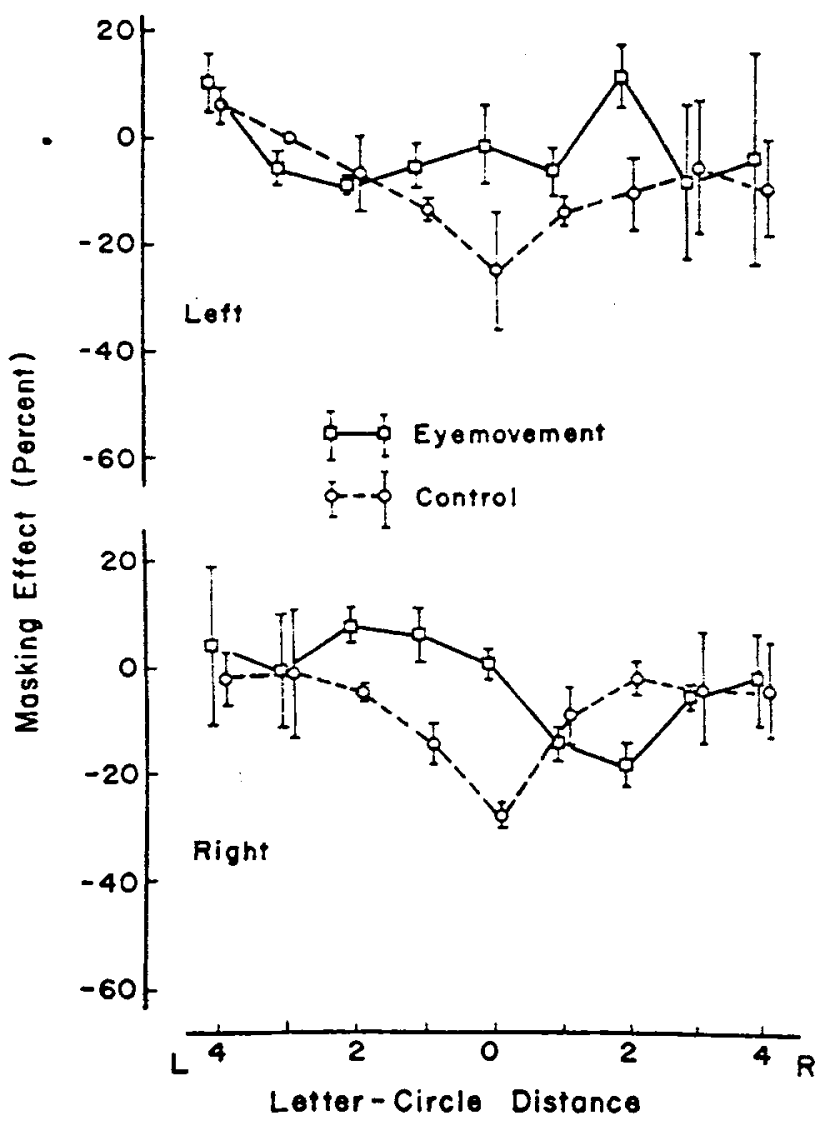

Fig. 6. Results of eye-movement experiment with metacontrast ring. (Same format as Fig. 5.)
Table 2

Percentage of Masks Reported at Various Locations

\begin{tabular}{|c|c|c|c|c|c|}
\hline \multirow{2}{*}{$\begin{array}{l}\text { Experimental } \\
\text { Condition* }\end{array}$} & \multicolumn{5}{|c|}{ Reported Minus Actual Locations } \\
\hline & -2 & -1 & 0 & 1 & 2 \\
\hline EM-L & 0.00 & 6.94 & 90.83 & 1.94 & 0.28 \\
\hline$C-L$ & 0.00 & 0.83 & 97.08 & 2.08 & 0.00 \\
\hline EMIR & 0.00 & 2.78 & 80.56 & 16.11 & 0.28 \\
\hline C-R & 0.00 & 0.00 & 97.08 & 2.92 & 0.00 \\
\hline
\end{tabular}

"EM-eye movement; $C$-control. $L, R$-left and right fixation on letters.

\section{PERCEIVED LOCATION AND PERSISTENCE OF STIMULI IN THE PRESENCE OF EYE MOVEMENTS}

\section{Perceived Mask Location}

In the masking experiment, Ss reported the apparent location of the mask as well as the identity of the letters. Table 2 summarizes the reported mask locations of all Ss in all trials and reveals veridical location perception, with only a slight tendency for the mask to appear displaced in the direction of the original fixation. On eye-movement trials, the mask is essentially never seen as coincident with the letter that it masks, which is two positions removed from the mask.

\section{Apparent Flash Persistence Through Eye Movements}

That a post-eye-movement mask appears to cover or surround one letter and yet suppresses the report of another raises the question of whether the letter perceived spatially coincident with the mask also appears to overlap it temporally. In the following experiment, Ss judged the location and persistence of two flashed stimuli with an eye movement intervening.

\section{Method}

Stimulus cards each had two figures occupying two of the positions occupied by letters in the masking experiment. Each trial involved two stimuli, one of which contained a square and a circle, and the other of which was composed of a vertical and a horizontal line segment. The stimuli from two typical trials would have appeared as shown in Fig. 7 had they been presented simultaneously; sometimes the lines were beside the closed figures, as in the upper example, and sometimes the two were coincident, as in the lower.

The experimental conditions were the same as in the eye-movement masking experiments, namely, the four conditions determined by the factors eye movement vs control and left vs right fixation on the first stimulus. The eye movement triggered the second of the two stimuli in the eye-movement conditions; the acceptable ISI window was $50-120 \mathrm{msec}$. Both stimuli were presented for $60 \mathrm{msec}$. Control-trial ISI was yoked to eye-movement trials.

Each of two Ss (A.O.D. and M.L.D.) received 16 trials in each condition; the trials were counterbalanced as regards closed figures vs lines first, circle vs square on left, vertical vs horizontal line on left, and figures overlapping vs figures interspersed (Fig. 7). The $\mathrm{S}$ reported (1) the spatial arrangement of the figures from left to right. as in the legend of Fig. 7. and 

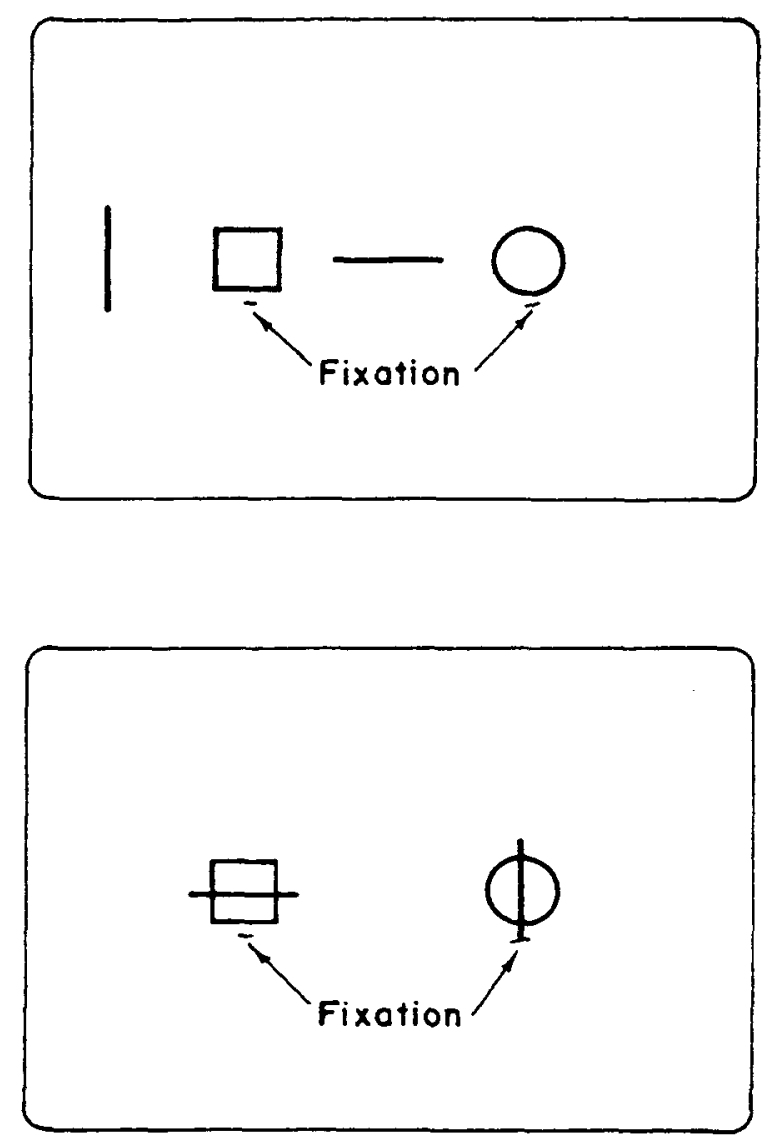

Fig. 7. Spatial arrangement of typical stimuli for fusion experiment. Upper stimulus-vertical line, square, horizontal line, circle. Lower stimulus-square with horizontal line, circle with vertical line.

(2) whether the two flashes appeared to be simultaneous, utterly distinct. or overlapping in time, i.e., were the first two figures still visible when the second were flashed (cf. Haber \& Standing. 1969).

\section{Results}

The perceived locations of the stimuli were veridical on all control trials and on 59 of the 64 eye-movement trials, the 5 exceptions involving reversal of the two figures (e.g., circle, square) in one stimulus. The two flashes never appeared to be simultaneous, owing no doubt to the minimum ISI of $50 \mathrm{msec}$. To judge whether the first persisted until the second began proved difficult, especially since but one succession appeared in a trial, unlike the experiment by Haber and Standing (1969). Nevertheless, $79 \%$ of the pairs seemed to overlap with ISIs of $50.85 \mathrm{msec}$, and only $30 \%$ with ISIs of 86-120 msec, a difference significant on the assumption of independent judgments (chi square calculated for each $\mathrm{S}$ and pooled $=23.38,2 \mathrm{df}$ ). Eye-movement and control conditions did not differ: $69 \%$ of all eye-movement trials and $64 \%$ of all control seemed to consist of temporally over lapping stimuli.

\section{SUMMARY AND DISCUSSION}

When an eye movement intervenes between a stimulus and a mask, the affect of the mask is to suppress the report of the portion of the stimulus that stimulates the same retinal receptors rather than the portion that is in the same position both objectively and apparently. The percept in Fig. 8 summarizes the data; the mask appears in its actual location (4), but masks the letter (2) in the same retinal location. Such masking is, however, weaker by half than masking without eye movements in otherwise comparable circumstances. There is no evidence that pattern and metacontrast masks differ in any of these respects.

The relevance of these results to normal visual functioning is limited by our presenting a brief stimulus immediately before an eye movement: the pre-eye-movement stimulus in normal conditions is typically available for at least $250 \mathrm{msec}$. Nor do the present results relate to whether a pre-eye-movement stimulus affects the perception of the post-eye-movement stimulus. A forward-masking study with mtervening eye movements is evidently needed, although such masking is likely weak in view of Turvey's (1973) data; A. O. Dick also has unpublished data from a similar. no-eye-movement condition showing little or no forward masking. The result that masking has upon retinally coincident stimuli encourages the speculation that, insofar as masking-like interaction is concerned, information from successive fixations is mutually disruptive rather than supportive. Indeed, the suppression of masking by the eye movement might be considered a countermeasure to aid in the collation of information from successive glances.

Detection experiments that involve eye movements suggest another interpretation of the suppression of masking: An eye movement may inhibit reception of the mask and therefore reduce its masking effect. Volkmann, Schick, and Riggs (1968) found that a stimulus normally just visible is visible only half the time if presented $75 \mathrm{msec}$ after the onset of a voluntary saccade. The suggestion that an eye movement suppresses visual perception generally would seem the most economical explanation of the fact (Gilbert, 1959) that successively presented phrases from text are easier to read when presented in the same spatial location than when presented side by side. One difficulty in comparing the detection and masking experiments is this: Volkmann, Schick, and Riggs found suppression of a stimulus presented $20 \mathrm{msec}$ before eye-movement onset, whereas stimulus report in the present experiment's

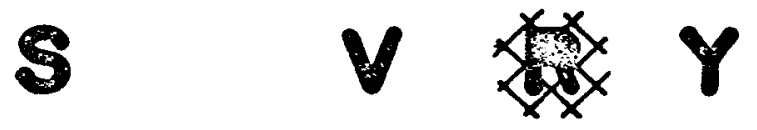

Fig. 8. Idealized percept consistent with results on typical EM-L trial. 
no-mask trials is as good when an eye movement follows (76\% correct) as when not $(79 \%$ correct). (The percentages reported are averages of the three Ss' percentages: the $3 \%$ difference is not statistically significant.)

When two pairs of suprathreshold geometric forms are presented with an intervening eye movement, the $S$ often reports that the first is still visible when the second is presented. and almost invariably reports veridically the location of the stimuli. That is, unlike whatever record of a stimulus is susceptible to masking, the apparent persistence of a pre-eye-movement stimulus remains fixed in space during the eye movement. It seems, therefore, that the visual mechanism that mediates perceptual report cannot be the same mechanism that preserves a stimulus while a mask overtakes it.

The present stimulus conditions are such that masking must certainly be "central" in Turvey's (1973) sense. That it is, nevertheless, afterimage-like suggests that the test-stimulus representation may be peripheral relative to the phenomenal representation. Alternatively, identification and localization of stimuli may occur in different mechanisms or information channels.

\section{REFERENCES}

Averbach. E.. \& Coriell. A. A. Short-term memory in vision. Bell System Technical Journal. 1961, 40. 309-328.
Bryden. M. P. The role of post-exposure eye movements in tachistoscopic perception. Canadian Journal of Psychology. 1961. 15, 220-225.

Crovitz, H. F., \& Davies, W. Tendencies to eye movements and perceptual accuracy. Journal of Experimental Psychology; 1962. 63, 495-498.

Eriksen. C. W., Collins, J. F., \& Greenspon. T. S. An analysis of certain factors responsible for nonmonotonic backward masking functions. Journal of Experimental Psychology. 1967. 75, 500-507.

Gilbert, L. C. Saccadic movements as a factor in visual perception in reading. Journal of Educational Psychology. $1959,50,15-19$.

Haber. R. N.. \& Standing, L. G. Direct measures of short-term visual storage. Quarterly Journal of Experimental Psychology. $1969,21,43-54$

Neisser. U. Cognitive psychology. New York: Appleton-Century-Crofts, 1967.

Smith, M. C., \& Scheller, P. H. Forward and backward masking: A comparison. Canadian Journal of Psychology, 1966, 20, 191-197.

Sperling. G. The information available in brief visual presentations. Psychological Monographs, 1960. 74, No. 11.

Turvey, M. T. On perceptual and central processes in vision: Inferences from an information-processing analy'sis of masking with patterned stimuli. Psychological Review, 1973, 80, 1-52.

Volkmann, F. C., Schick, A. M. L., \& Riggs, L. A. Time course of visual inhibition during voluntary saccades. Journal of the Optical Society of America, 1968, 58, 562-569.

(Received for publication December 18, 1972: revision received March 30, 1973.) 